\title{
Elimination Of Right Superior Pulmonary Vein Firing During Radiofrequency Ablation Around the Left Pulmonary Veins: What is the Mechanism?
}

\author{
AURELIE GUIOT, M.D., TIMIR BAMAN, M.D., FRED MORADY, M.D., \\ and AMAN CHUGH, M.D.
}

From the Section of Electrophysiology, Division of Cardiovascular Medicine, University of Michigan, Ann Arbor, Michigan, USA

autonomic nervous system, atrial fibrillation, catheter ablation, ganglionated plexus, pulmonary veins

A 74-year-old man with a history of drug-refractory paroxysmal atrial fibrillation (AF) for 10 years was referred for catheter ablation. His echocardiogram showed normal left ventricular ejection fraction and left atrial (LA) diameter. He presented to the electrophysiology laboratory in si-

J Cardiovasc Electrophysiol, Vol. 23, pp. 442-444, April 2012.

Supported in part by a grant from the Leducq Transatlantic Network.

No disclosures.

Address for correspondence: Aman Chugh, M.D., Cardiovascular Center, SPC 5853, 1500 E. Medical Center Dr., Ann Arbor, MI 48109-5853, USA. Fax: 734-936-7026; E-mail: achugh@umich.edu

doi: 10.1111/j.1540-8167.2011.02113.x nus rhythm. After transseptal puncture, a replica of the LA was created using a 3D mapping system (CARTO, Biosense Webster, Diamond Bar, CA, USA) and an irrigated-tip ablation catheter (Thermocool, Biosense Webster). The left-sided pulmonary veins (PVs) were first targeted. To minimize interaction between the ablation and the ring catheters (Lasso, Biosense Webster), the latter was placed in the right superior PV (RSPV) during antral ablation of the left-sided PVs. Shortly after commencing with radio frequency (RF) ablation around the left PVs, a late potential appeared $200 \mathrm{~ms}$ following the RSPV potential (Fig. 1). First, there was a 2:1 relationship between the RSPV potential and the late potential, and after further ablation around the left antrum, there was a 1:1 relationship. As we continued ablating around the left antrum, and the crux between the left superior and inferior PVs, the late potential would appear to be eliminated, only to reappear immediately after cessation of RF

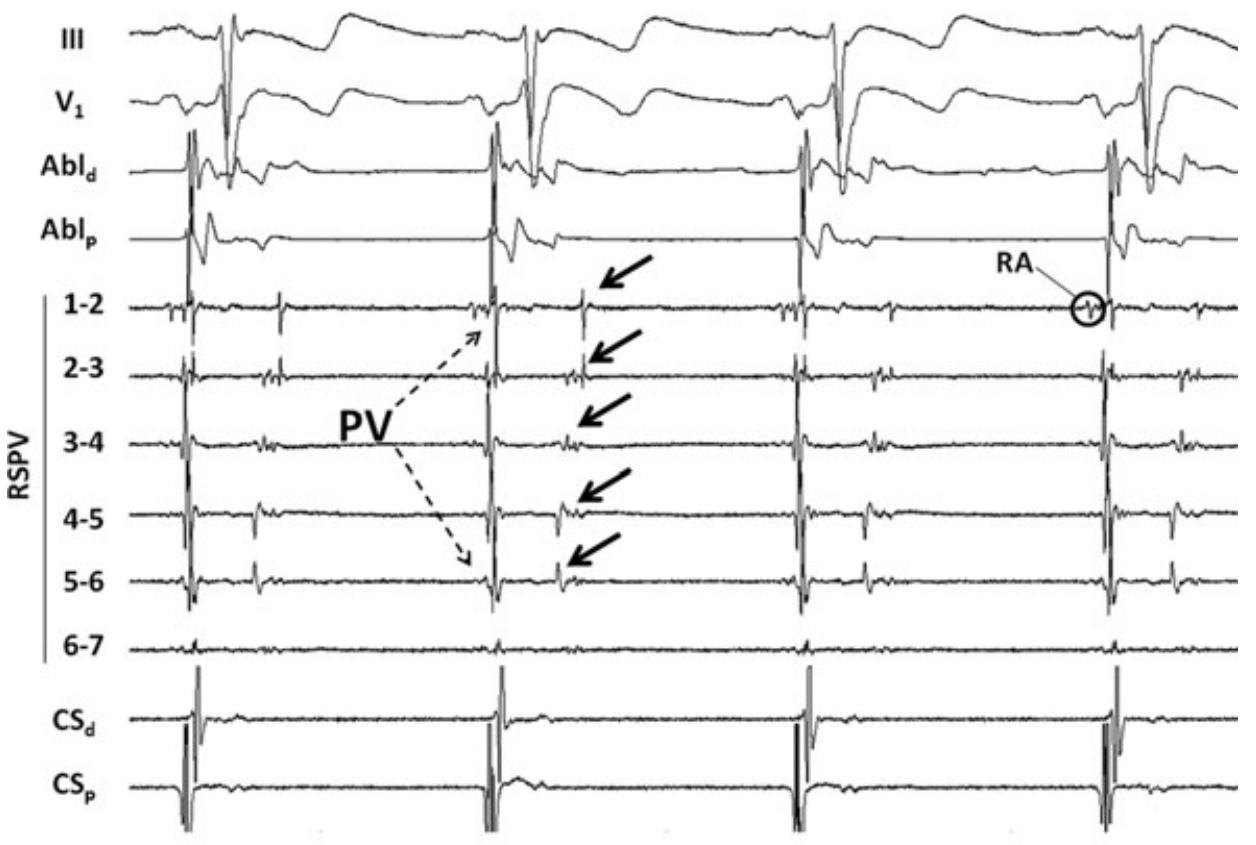

$500 \mathrm{~ms}$
Figure 1. During radiofrequency $(R F)$ energy delivery around the left-sided pulmonary veins $(P V)$, a late potential appeared (arrows) on the recording by the ring catheter in the right superior $P V(R S P V) . R A=$ far field right atrial electrogram. 
Figure 2. During RF energy delivery at various sites around the left-sided PVs, the late potential (red arrows) would appear to be eliminated. This usually coincided with sinus slowing or arrest, suggesting an autonomic mechanism. Note that after a 6-second pause, the potential reappears (orange arrow), consistent with firing from the RSPV, resulting in an atrial premature depolarization.

\section{A}

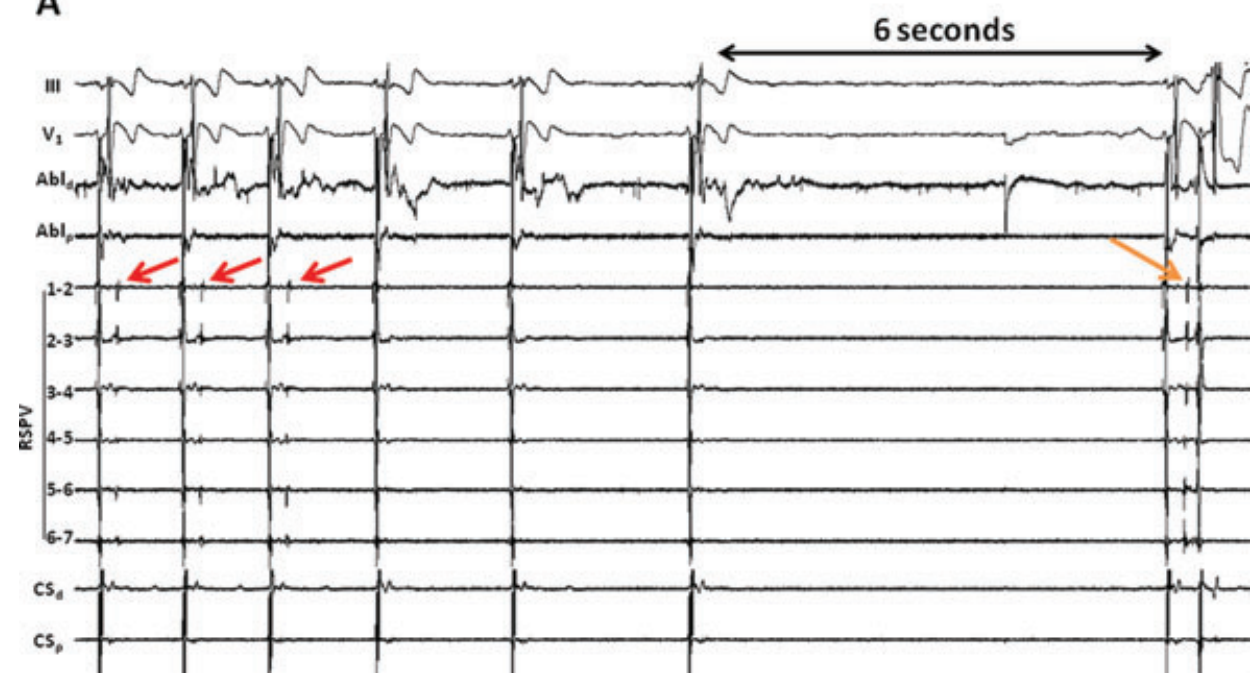

energy delivery (Fig. 2). Frequently, but not always, the transient elimination of the late potential coincided with sinus slowing or pause (Fig. 2). Conversely, the reappearance of the late potential coincided with acceleration of the sinus rate. The late potential in the RSPV was permanently eliminated during RF energy delivery outside the left inferior PV (Fig. 3A,B). What is the mechanism of elimination of the late potential in the RSPV during RF energy delivery outside the left inferior PV?

\section{Discussion}

When RF ablation results in unexpected consequences remote from the target site, there may be 3 possible explanations. First, it is plausible that a myocardial connection between the target and the remote sites may have been eliminated. ${ }^{1,2}$ Second, it is possible that the vascular supply to the remote site may have been disrupted. Finally, owing to the interconnections between the various ganglionated plexi (GP), ${ }^{3,4}$ the cardiac intrinsic autonomic nervous system (IANS) may be involved. There are several observations that support an autonomic mechanism in the current case. First, the elimination of the late potential in the RSPV frequently coincided with vagally mediated sinus slowing. Second, the reappearance of the potential following discontinuation of RF energy coincided with recovery from vagal stimulation, that is, acceleration of the sinus rate. Also, the site where
RF energy delivery led to permanent elimination of the late potential is consistent with the location of the inferior left GP.

It is unlikely that these observations are due to ablation of a long myocardial connection, for example, a shared fascicle, between the left- and right-sided PVs. If this were the case, one would expect to see elimination or at least a delay of conduction into the RSPV, which was not observed (Fig. 2). The fact that the second potential only appeared after initiation of RF energy delivery is incompatible with the presence of a shared fascicle, which implies an anatomical connection. An interruption of the vascular supply to the RSPV would also be expected to result in delay or elimination of conduction.

In animal models, focal firing from a PV has been demonstrated during stimulation of an ipsilateral GP. ${ }^{4}$ It may be unexpected to observe this phenomenon during stimulation, due to RF energy delivery in the current case, of the contralateral GP. It is likely that appearance of the late potential represents firing from the RSPV, induced by stimulation of the left-sided GPs. It follows then, after ablation of the inferior left GP, RSPV firing could no longer be induced, supporting the presence of neural connections between contralateral GPs.

Stimulation of the GPs typically results in rapid, repetitive discharges from the PVs. ${ }^{4}$ In the current case, only a single discharge was observed from the RSPV. It is possible that
Figure 3. A: A $3 D$ map of the left atrium showing the ablation sites (red tags) required for $P V$ isolation (posteroanterior view). The pink tags represent sites where $R F$ energy delivery either temporarily or permanently (yellow arrow) eliminated firing from the RSPV. The orange tag denotes the site where RF ablation isolated the left-sided PVs. LS = left superior; $L I=$ left inferior; $R I=$ right inferior. $B$ : Cinefluoroscopic view showing the position of the ablation (arrow) and ring catheters at the time when RF energy delivery outside the LIPV led to permanent elimination of RSPV firing. CS $=$ coronary sinus.
A

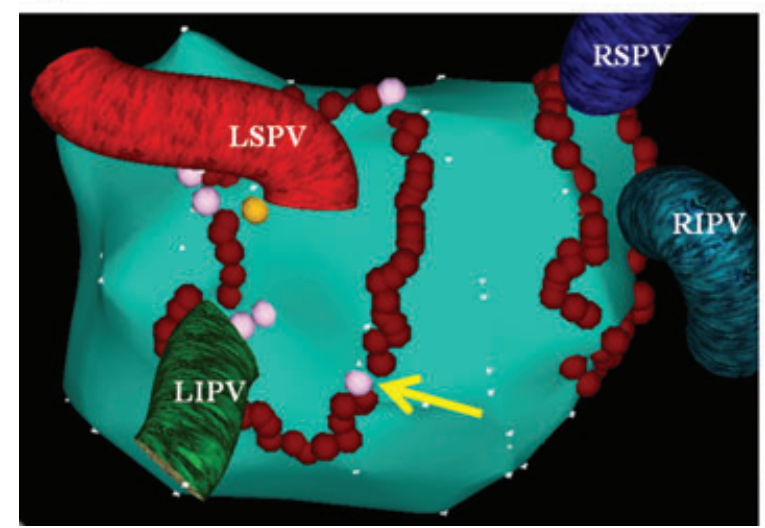

B

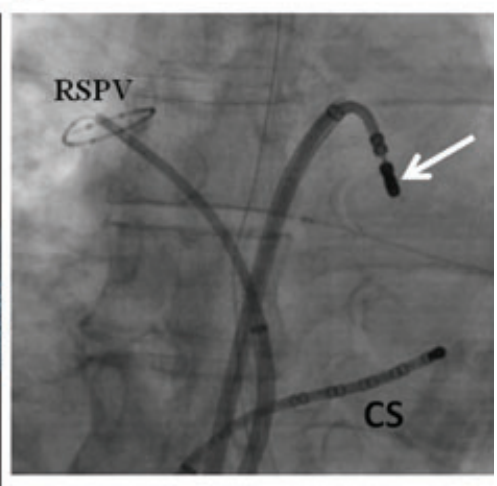


the electrophysiologic milieu at the LA/PV junction was not conducive to a more sustained arrhythmia. This is supported by the fact that the focal discharge from the RSPV was incapable of conduction into the LA, likely owing to prolonged refractoriness. However, at a time when the atrial effective refractory period would be expected to have significantly shortened, for example during GP stimulation as shown in Figure 2, conduction out of the RSPV was observed, resulting in an atrial premature depolarization. It is also possible that the antifibrillatory effect of ablation of the left-sided GPs may have made it less likely for the RSPV to generate repetitive firing and $\mathrm{AF}$.

These findings are noteworthy for a number of reasons. First, they confirm findings from animal studies that ablation of a particular GP not only suppresses rapid firing from the ipsilateral PVs but also contralateral PVs. ${ }^{4}$ These observations also support a strategy of isolation of all PVs as opposed to isolation of only the "culprit" vein. A strategy of targeting all PVs may improve outcomes by ren- dering PVs less arrhythmogenic by means of partial GP ablation.

\section{References}

1. Chan CP, Wong WS, Pumprueg S, Veerareddy S, Billakanty S, Ellis C, Chae S, Buerkel D, Aasbo J, Crawford T, Good E, Jongnarangsin K, Ebinger M, Bogun F, Pelosi F, Oral H, Morady F, Chugh A: Inadvertent electrical isolation of the left atrial appendage during catheter ablation of persistent atrial fibrillation. Heart Rhythm 2010;7:173-180.

2. Perez-Castellano N, Villacastin J, Salinas J, Vega M, Moreno J, Doblado M, Ruiz E, Macaya C: Epicardial connections between the pulmonary veins and left atrium: Relevance for atrial fibrillation ablation. J Cardiovasc Electrophysiol 2011;22:149-159.

3. Hou Y, Scherlag BJ, Lin J, Zhou J, Song J, Zhang Y, Patterson E, Lazzara R, Jackman WM, Po SS: Interactive atrial neural network: Determining the connections between ganglionated plexi. Heart Rhythm 2007;4:5663.

4. Lu Z, Scherlag BJ, Lin J, Yu L, Guo JH, Niu G, Jackman WM, Lazzara R, Jiang H, Po SS: Autonomic mechanism for initiation of rapid firing from atria and pulmonary veins: Evidence by ablation of ganglionated plexi. Cardiovasc Res 2009;84:245-252. 